\title{
Red cell distribution width: A novel inflammatory marker in clinical practice
}

We intentionally read the article "Red cell distribution width is associated with acute myocardial infarction in young patients" written by Uysal et al. with great interest [1]. They aimed to investigate the relationship between red cell distribution width (RDW) and acute ST-elevated myocardial infarction (STEMI) in young patients. Compared with young patients with normal coronaries, young patients with STEMI had significantly higher values of RDW. Higher levels of RDW was an independent predictor of STEMI in young patients together with gender, history of hyperlipidemia. The study is successfully designed and presented. We believe that these findings will elucidate further studies about RDW as a surrogate marker of inflammation and atherosclerosis. Thank to the authors for their contribution.

RDW, a measurement of variability and size of erythrocytes, can be easily measured during routine complete blood counts [2]. Increased RDW, independent of hemoglobin values, has been demonstrated to be associated with negative clinical outcomes in patients with heart failure, previous myocardial infarction, and stable coronary artery disease [3]. The association of RDW with adverse outcomes in cardiovascular diseases has not been fully understood. Inflammation may cause changes in red blood cell maturation by disturbing the red cell membrane, leading to increased RDW.

The pathogenesis of atherosclerosis is multifactorial; however, it is considered to be an inflammatory disease [4]. Previous studies have demonstrated that elevated levels of inflammatory molecules are markers of atherosclerotic disease activity and also indicate an increased risk for the progression of atherosclerosis. So, there is a significant relationship between the endothelial dysfunction, and inflammatory parameters and cardiovascular risk factors such as hypertension, diabetes mellitus, smoking and hypercholesterolemia [5]. Because these factors may affect the levels of RDW, compared to patient groups, control groups should be similar in features according to risk factors and in terms of the number of the individuals. If we see an increased RDW level in a patient, we do think that there is a problem about hematologic parameters or the patients may have some of these diseases. Although it is an easy method to assess inflammation and it may be useful in clinical practice, RDW itself alone without other inflammatory markers may not give information to clinicians about the endothelial inflammatory condition of the patient. So, we think that it should be evaluated together with other serum inflammatory markers.

In addition, the authors are required to give information about the selection of patients in the control group. Finally, all measurements were performed 30 min after blood collection in this study. They should define why they measured after $30 \mathrm{~min}$ because 30 min delay after blood sampling can cause abnormal results in RDW measurement. In addition, these patients are expected to have higher inflammatory marker levels compared to healthy populations. Further studies should be made to enlighten the role of RDW as an inflammatory marker in atherosclerotic disease subjects.

\section{Conflict of interest: none declared}

\section{References}

1. Uysal OK, Duran M, Ozkan B et al. Red cell distribution width is associated with acute myocardial infarction in young patients. Cardiol J, 2012; 19: 597-602.

2. Tonelli M, Sacks F, Arnold M, Moye L, Davis B, Pfeffer M. Relation Between red blood cell distribution width and cardiovascular event rate in people with coronary disease. Circulation, 2008; 117: $163-168$.

3. Felker G, Allen L, Pocock S et al. Red cell distribution width as a novel prognostic marker in heart failure: data from the CHARM Program and the Duke Databank. J Am Coll Cardiol, 2007; 50: 40-47.

4. Tatli E, Aktoz M, Buyuklu M, Altun A. The relationship between coronary artery disease and uric acid levels in young patients with acute myocardial infarction. Cardiol J, 2008; 15: 21-25.

5. Isik T, Ayhan E, Uyarel $\mathrm{H}$ et al. Increased mean platelet volume associated with extent of slow coronary flow. Cardiol J, 2012; 19: 355-362.

Sait Demirkol', Sevket Balta ${ }^{1}$, Mustafa Cakar ${ }^{2}$, Murat Unlu $^{1}$, Zekeriya Arslan ${ }^{1}$, Ugur Kucuk ${ }^{1}$

${ }^{1}$ Department of Cardiology, Gulh ane Medical Academy, Tevfik Saglam St., 06018 Etlik-Ankara, Turkey tel: +90 312 3044264, fax: +90 312-3044250, e-mail: saitdemirkol@yahoo.com ${ }^{2}$ Department of Internal Medicine, Gulhane Medical Academy, Ankara, Turkey 